\title{
GPS-based Interferometric Heading Determination Using Carrier Phase Measurement
}

\author{
Jhabindra Khanal ${ }^{1}$, Felipe P. Vista $\mathrm{IV}^{2}$ and Kil To Chong ${ }^{3, *}$

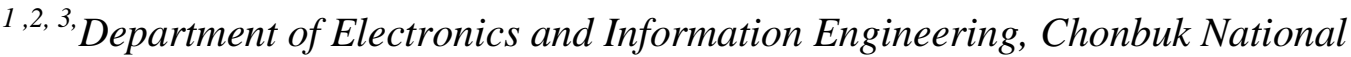 \\ University, South Korea \\ ${ }^{1}$ E-mail:Jhabindra@jbnu.ac.kr (Khanal) \\ ${ }^{3}$, E-mail:kitchong@jbnu.ac.kr(Chong)
}

\begin{abstract}
This paper presents a determination of a heading angle using carrier phase differential GPS (CPDGPS) measurement between two slave antennas. Heading of a platform can be obtained from measurements with two linearly independent interferometers where each interferometer involves of two separate antennas or baseline length. In this particular test, Such antennas are separated by a small length to remove the corresponding errors formed by the GPS system, such technique of removing particular errors are named as Single Difference(SD) and Double Difference $(D D)$ Measurements. Measuring phase difference between antennas is a quite complex method in GPS because of the full number of carrier cycles are unknown or it is ambiguous in nature known as integer ambiguity problem. To remove the integer ambiguity problem we processed the LAMBDA (Least Square Ambiguity Decorrelation Adjustment) to obtain a higher heading accuracy for static experiments. Furthermore, the relative position of a rover receiver is also mentioned in the paper within a centimeter level of accuracy.
\end{abstract}

Keywords: GPS, double difference, RINEX, least square, heading determination

\section{Introduction}

GPS interferometry is a technique that is based on the measurement of the phase difference of the GPS carrier signal measured at two or more antennas. Pseudo-range and carrier phase are the two basic range measurement in GPS system. The purpose of using carrier phase is to have a precise measurement of the signal phase difference. Currently, in a GPS distance measurement system mostly either L1, L2 or a combination of both signals is used. The wavelengths of the signals are about $20 \mathrm{~cm}$. If we track the signal properly the alignment error of the receiver reduces within one percent of the wavelength. Besides for pseudorange-code measurement, the length of the code is about $293 \mathrm{~cm}$ and the alignment error is higher, hence the carrier phase measurement is more precise than code measurement. Attitude determination for short distance, Zero baseline, and two baselines can be found in [1-4]. Differential carrier phase GPS based heading determination is used, alternative to expensive Inertial Measurement Units (IMU's) since IMU's require highly accurate accelerometer and gyros to determine attitude (roll, yaw and pitch).

The problem of processing Carrier Phase (CP) technique in GPS is quite complex since a full number of carrier cycles are unknown initially. The positive thing is that the fractional part of the signals can be measured successively. To solve the integer ambiguity problem the LAMBDA algorithm can be implemented because of its successive nature at any condition [5]. DD technique differences the carrier phase observation equation two times firstly between antennas and between satellites, to remove the bias due to satellite and receiver clock offsets. The exact truth reference is not available for heading 
comparison. The headings obtained from the different experiments are compared to the Google Earth [6].

In this paper GPS- based interferometry heading determination using carrier phase has been presented. Stationary experiments have been carried out and different sets of data in the binary format were collected from both receivers and later converted to the Receiver Independent Exchange (RINEX) format and post processed. To precise measure of heading angle between two GPS receivers, the baseline vector between two receivers is a core process. The core algorithm or least squares (LS) minimization is used. To solve integer ambiguity the LAMBDA has been processed. We also calculated the position of a rover receiver with respect to a stationary receiver in Earth center Earth Fixed (ECEF) frame. For heading purpose ECEF frame has been converted to the navigation North East and Up (NEU) frame.

\section{Key Technique of the Phase Measurement and Error Reduction}

\subsection{Carrier Phase Measurement}

Carrier phase is a measurement which calculates the phase difference between the phase of the receiver-generated carrier signal at the reception time interval and the phase of the carrier signal generated by the satellite at the transmission time interval. Because of the short wavelength of the GPS carrier signal (e.g. 0.19m for GPS L1 signal), error in the measurement of phase leads to only a small error in the range measurement. Hence, carrier phase range evaluation is a lot accurate compared to code range evaluation. This integer number is a bias in the range measurement and is called the integer ambiguity. The carrier phase observation ( $\left.\phi_{s}^{i}\right)$ is given below.

$$
\Phi_{s}^{i}=r_{s}^{i}+\lambda N_{s}^{i}+T_{s}^{i}-I_{s}^{i}+\beta^{i}+\beta_{s}+\varepsilon_{s \phi}^{i}
$$

Where the subscript $s$ denotes the base receiver and superscript $i$ denotes the $i^{i^{\text {th }}}$ satellite. ${ }^{r_{s}^{i}}$ is the range between the base receiver and $i^{i t h}$ satellite. Ionosphere delay is symbolized by $I . T$ refers for Troposphere delay, satellite clock error is $\beta^{i}, \beta_{s}$ is the receiver clock bias, ${ }_{s, \phi}$ is the carrier phase measurement error. The term $N$ is an integer ambiguity with the corresponding wavelength of $\lambda$.

\subsection{Single Difference}

Relative positioning applications consist of two GPS receivers, one GPS receiver is termed as a base station, other GPS receiver is termed as a rover. The convention following the above naming process doesn't illustrate that the base station is static, in turn, it illustrates that the rover's location comparative to the reference station is highly important. For example, to evaluate the location of an aircraft, a static GPS antenna/receiver pair is located in a specific test area as a reference station. The aircraft containing a GPS antenna/receiver pair is capable of communicating with the ground reference station and calculating its position relative to the ground reference station. These two receivers are affected by identical satellite clock errors. This identical satellite clock error can be eliminated if a measurement difference is taken with respect to the same satellite. This is known as the single difference (SD) technique. Adding one more rover receiver $r$ to form the single difference are shown in equation (2).

$$
\begin{gathered}
\Delta \phi_{s, r}{ }^{i}=\phi_{s}^{i}-\phi_{r}^{i} \\
\text { Or, } \phi_{s}^{i}-\phi_{r}^{i}=r_{s}^{i}-r_{r}^{i}+\lambda N_{s}^{i}-\lambda N_{r}^{i}+T_{s}^{i}-T_{r}^{i}-I_{s}^{i}-I_{r}^{i} \\
+\beta^{i}-\beta^{i}+\beta_{s}^{i}-\beta_{r}^{i}+\varepsilon_{\mathrm{s}, \phi}^{i}-\varepsilon_{r, \phi}^{i}
\end{gathered}
$$




$$
=\Delta r_{s, r}^{i}+N_{s, r}^{i}+\Delta \beta_{s, r}^{i}-\Delta I_{s, r}^{i}+\Delta T_{s, r}^{i}+\varepsilon_{s, r, \phi}^{i}
$$

Where, ${ }^{\Delta r_{s, r}^{i}}=r_{s}^{i}-r_{r}^{i}$ and so on. $\Delta$ Denotes the single difference. Superscript $i$ is a satellite.

\subsection{Double Difference}

The terms $\Delta I_{s, r}^{i}$ and $\Delta T_{s, r}^{i}$ represent the ambiguities produced by atmosphere decorrelation between the base and the rover receiver. The term ${ }^{\beta_{s, r}^{i}}$ represents the clock error difference between the base station and the rover which is similar to all of the satellites. If a further difference (two single difference) is taken for single difference evaluation corresponding to the satellite, this common clock difference error can be eliminated. This is known as the double difference (DD) technique. The double difference measurements are given by:

$$
\begin{gathered}
\nabla \Delta \phi_{s, r}^{i, j}=\Delta \phi_{s, r}^{i}-\Delta \phi_{s, r}^{j} \\
\nabla \Delta \phi_{s, r}^{i, j}=\Delta r_{s, \mathrm{r}}^{i}-\Delta r_{\mathrm{s}, \mathrm{r}}^{j}+\Delta N_{s, r}^{i}-\Delta N_{s, r}^{j}+\Delta I_{\mathrm{s}, \mathrm{r}}^{i}-\Delta I_{\mathrm{s}, \mathrm{r}}^{j} \\
+\Delta \beta_{s r}-\Delta \beta_{s r}+\Delta T_{s, r}^{i}-\Delta T_{s, r}^{j}+\Delta \varepsilon_{s, r}^{i}-\Delta \varepsilon_{s, r, \phi}^{j} \\
\nabla \Delta \phi_{s, r}^{i, j}=\nabla \Delta r_{s, r}^{i, j}+\nabla \Delta N_{s, r}^{i, j}-\nabla \Delta I_{s, r}^{i, j}+\nabla \Delta T_{s, r}^{i, j}+\nabla \Delta \varepsilon_{s, r, \phi}^{i, j}
\end{gathered}
$$

Where the parameters are denoted with a double difference as the prefix (e.g. Double difference integer ambiguity, double difference carrier phase noise).

If we considered that the base station is relatively near to the rover, the errors produced by the atmosphere decorrelation can be overlooked. This gives the simplest double difference measurements as:

$$
\nabla \Delta \phi_{s, r}^{i, j}=\nabla \Delta r_{s, r}^{i, j}+\nabla \Delta N_{s, r}^{i, j}+\nabla \Delta \varepsilon_{s, r, \phi}^{i, j}
$$

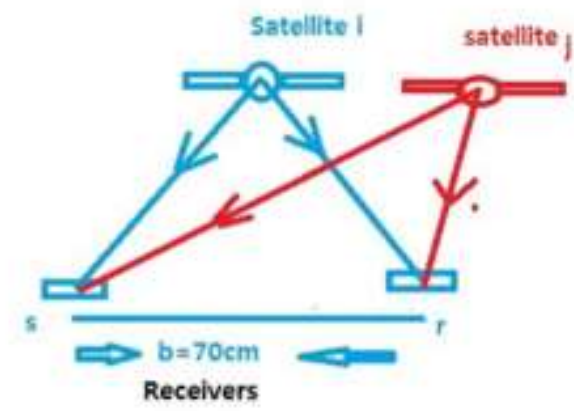

Figure 1. DD Measurement

The single difference can be written as the line of sight vector $u$ to the inner product of baseline vector $\boldsymbol{b}$ [7]. The noise term will be dropped to simplify the expression. Hence the equation (8) becomes,

$$
\begin{aligned}
& \nabla \Delta \phi_{s, r}^{i, j}=\left(u^{i}-u^{j}\right) \cdot b_{s r}+\nabla \Delta N_{s, r}^{i, j} \\
& \nabla \Delta \phi_{s, r}^{i, j}=u_{s r}^{i j} \cdot b_{s r}+\nabla \Delta N_{s, r}^{i, j}
\end{aligned}
$$

The rest of the parameters to be computed are the baseline vector $\boldsymbol{b}$ which consists of three components $\left(b_{x}, b_{y}, b_{z}\right)$ and an unknown integer carrier cycle ambiguity $N$. Two satellites are needed to form each DD. Thus, in order to form four DD equations, a 
minimum of five satellites are necessary. The expanded matrix form of the equation (10) for four DDs with 5 satellites (out of them one satellite is chosen as reference which has higher elevation angle) to form DDs.

$$
\left[\begin{array}{l}
\nabla \Delta \phi_{s r, 1}^{i j} \\
\nabla \Delta \phi_{s r, 2}^{i j} \\
\nabla \Delta \phi_{s r, 3}^{i j} \\
\nabla \Delta \phi_{s r, 4}^{i j}
\end{array}\right]_{=}\left[\begin{array}{ccc}
u_{x}^{12} & u_{y}^{12} & u_{z}^{12} \\
u_{x}^{13} & u_{y}^{13} & u_{z}^{13} \\
u_{x}^{14} & u_{y}^{14} & u_{z}^{14} \\
u_{x}^{15} & u_{y}^{15} & u_{z}^{15}
\end{array}\right]\left\{\begin{array}{l}
b_{x} \\
b_{y} \\
b_{z}
\end{array}\right\}_{+}\left\{\begin{array}{l}
N_{1} \\
N_{2} \\
N_{3} \\
N_{4}
\end{array}\right\} \lambda
$$

Where $\nabla \Delta \phi_{s r, 1}^{i j}$ is the first of four DDs, $u^{12}$ represents the differenced unit vector or line of sight vector between the two satellites under consideration, $\boldsymbol{b}$ is the baseline vector, $\mathrm{N}$ is the associated integer carrier cycle ambiguity, and $\lambda$ is the corresponding wavelength. Equation (11) can be simplified and written in the following form.

$$
y=H b+N
$$

\subsection{Least Square Method}

To minimize float solution by using least squares, In equation (12) once the $N$ is found the $\boldsymbol{b}$ can be found easily. The minimization gives the float $\hat{b}$ and $\widehat{N}$ as real numbers set with their variance-covariance Matrix $\sigma_{\tilde{N}}$, it comes from the ordinary normal solution from the equation (13).

$$
[H]^{T} \sum_{y}^{-1}[H][\widehat{b}, \widehat{N}] T=H^{T} \sum_{y}^{-1} y
$$

The output from the equation (13) are,

$$
\begin{aligned}
& b_{\text {float }}=b_{x}, b_{y}, b_{z} \\
& \widehat{N}_{\text {real }}=\left(\begin{array}{l}
\widehat{N}_{1} \\
\widehat{N}_{2} \\
\widehat{N}_{3} \\
\widehat{N}_{4}
\end{array}\right) \\
& \sigma_{\hat{N}}=\left[\begin{array}{llll}
\sigma_{\hat{N}(1,1)} & \sigma_{\hat{N}(1,2)} & \sigma_{\hat{N}(1,2)} & \sigma_{\hat{N}(1,4)} \\
\sigma_{\hat{N}(2,1)} & \sigma_{\hat{N}(2,2)} & \sigma_{\hat{N}(2,3)} & \sigma_{\hat{N}(2,4)} \\
\sigma_{\hat{N}(3,1)} & \sigma_{\hat{N}(3,2)} & \sigma_{\hat{N}(3,3)} & \sigma_{\hat{N}(3,4)} \\
\sigma_{\hat{N}(4,1)} & \sigma_{\hat{N}(4,2)} & \sigma_{\hat{N}(4,3)} & \sigma_{\hat{N}(4,4)}
\end{array}\right]
\end{aligned}
$$

LAMBDA takes the values (real integer values and variance-covariance matrix) from the above minimization process as input to fix real values to an integer set. After getting integer values from the LAMBDA we process to fix solution. The float solution stands for the float baseline values from the least square method which is not more accurate so the back substitution requires fixing the values. The fixed baseline vector can be obtained as follows.

$$
b_{\text {fixed }}=\left(\mathrm{H}^{T} \mathrm{H}\right)^{-1} \mathrm{H}^{T}(\nabla \Delta \phi-N)
$$

\subsection{Heading Determination}

In a twin receiver system, the origin is at the antenna $s$. Both the heading and the pitch are defined by the baseline from antenna $s$ to antenna $r$. The three axes of the ENU coordinate system are East, North, Up. East axis points to East. North axis Points to North, and Up axis points upward to the local vertical. This is especially true when performing the attitude determination using the pointing system. The Azimuth angle is also defined in ENU coordinates. It is positive in the clockwise direction from the North, and negative in 
the counter-clockwise direction. The elevation angle is also defined in the local level system with a positive angle above the local level plane and negative angles below it. In some instances, the true heading direction of the vehicle may be the same as the baseline formed by two antennas. If this is the case, the GPS-determined heading is equal to the heading of the Platform. That is why the misalignment angle between the two headings needs to be calculated and taken into account [8].

As shown in the figure below, the heading is the rotation angle about the z-axis (up), clockwise being positive pitch is the rotation angle about the rotated $\mathrm{x}$-axis (east). Where “ $\Delta N, \Delta E$ and $\Delta U$ are the North East and Up baseline components in local Navigation(NEU) coordinate system can be measured directly from the carrier phase positioning System.

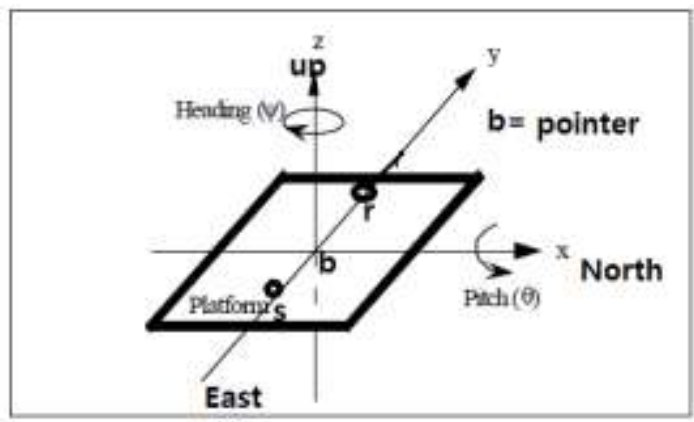

Figure 2. Heading Platform of Two Receivers

The fixed baseline vector from the equation (15) is given in Earth Center Earth Fixed (ECEF) frame. For navigation purpose, we have converted to North, East, and Up (NEU) frame.

$$
\left[\begin{array}{l}
\Delta E \\
\Delta N \\
\Delta U
\end{array}\right]_{E N U}=\left[\begin{array}{ccc}
-\sin \lambda & \cos \lambda & 0 \\
-\cos \lambda \sin \varphi & -\sin \lambda \sin \varphi & \cos \varphi \\
\cos \lambda \cos \varphi & \sin \lambda \cos \varphi & \sin \varphi
\end{array}\right]\left[\begin{array}{l}
b_{x} \\
b_{y} \\
b_{z}
\end{array}\right]_{E C E F}
$$

Where, $\lambda$ and ${ }^{\varphi}$ are the latitude and longitude of the stationary receiver $s$, then the heading and baseline length can be obtained using following formulae.

$$
\begin{aligned}
\text { Heading } \psi & =\arctan \Delta E / \Delta N(\mathrm{rad}) \\
\text { Baseline length } b & =\sqrt{\Delta N^{2}+\Delta E^{2}+\Delta U^{2}} \text { (Meter) }
\end{aligned}
$$

Position of the rover receiver $=$ position of the base receiver + baseline vector $(\boldsymbol{b})$

\section{Software and Hardware}

The Ublox EVK-M8T product outputs both code data and phase data in a binary format (i.e. UBX proprietary format) for the L1 frequency that allows to compute highly accurate range solutions. First, we assumed that the receiver $s$ is the stationary receiver whose position has been pre-surveyed. $r$ is the rover receiver whose position has to be determined.

We collected the data from both receivers at the same time for all observed epochs. The Ublox provides the free software to log the data simultaneously [9]. All the observation data were generated at a recording rate of $1 \mathrm{HZ}$ in binary format with an elevation cut-off angle of 10 degrees. Binary logged file can be converted by using RTKLIB [10] to create Receiver Independent Exchange Format (RINEX) files [11]. RINEX is a data interchange format for binary raw satellite navigation system data. The RINEX file outputs the Observation files and Navigation files. The Navigation file has 
created for positions of each satellite and forming double difference for corresponding two GPS receivers. The code has been written in MATLAB environment. The USB cable is capable for both power supply and data logging. The data logger is shown in Figure (3).

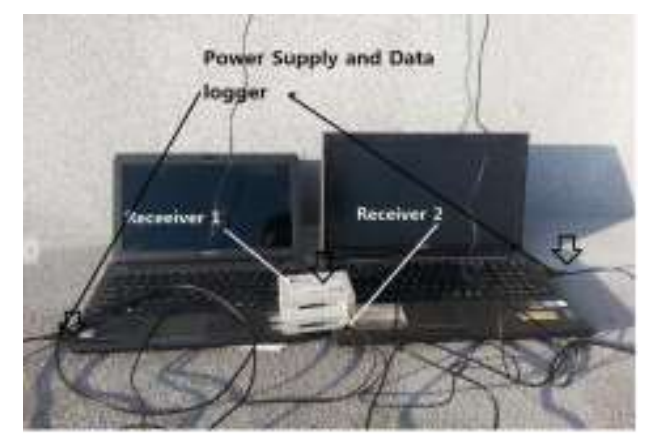

Figure 3. Data Logger and Receivers

\section{Experimental Setup and Results}

For the experiment, two different setups have been made at the ground of Chonbuk National University, Jeonju, Korea, The baseline length is set to $0.70 \mathrm{~m}$ for both experiments. The possibility of multipath, signal block and gross error have almost been ignored. The two GPS antennas are mounted in the West to East (but not exactly) direction. And another setup is performed in the path of the university ground pointing towards South to North direction.

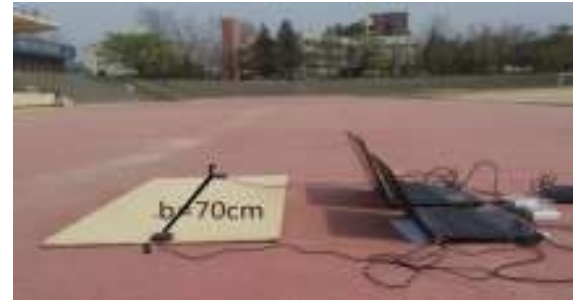

Figure 4. Exp. A, East to West

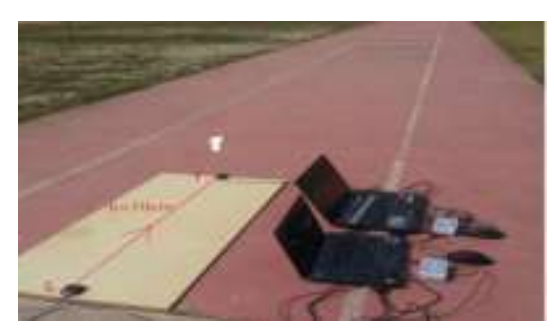

Figure 5. Exp. B, South to North

\subsection{Carrier Phase SD and DD Performance}

For raw phase measurements of tracked satellites, we have chosen satellites, according to the higher elevation angle. The higher elevation angle gives less possibility of multipath. For experiment A, both the master and rover receiver tracked ten satellites, out of them common satellites were Sv16, Sv26, Sv27, Sv29 and Sv31 with higher elevation angle $53.4090^{\circ}, 71.4680^{\circ}, 23.2180^{\circ}, 22.5220^{\circ}$ and $57.7270^{\circ}$, respectively. Sv 26 with $71.4680^{\circ}$ elevation angle was chosen as a reference to create a single and double difference. The raw measurement of carrier cycles is given in Figure (6). 


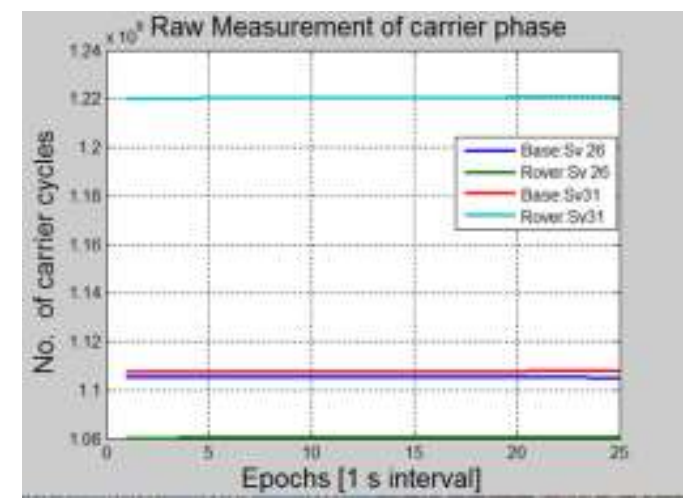

Figure 6. CP Measurement for Sv 26 and Sv 31

In Figure (6) the carrier phase is measured for sv 26 and sv 31 from both receives at the same time in cycles and the epoch interval is one second. SD for receiver $\mathbf{s}$ and $\mathbf{r}$ with respect to satellite sv26 is shown in Figure 7.

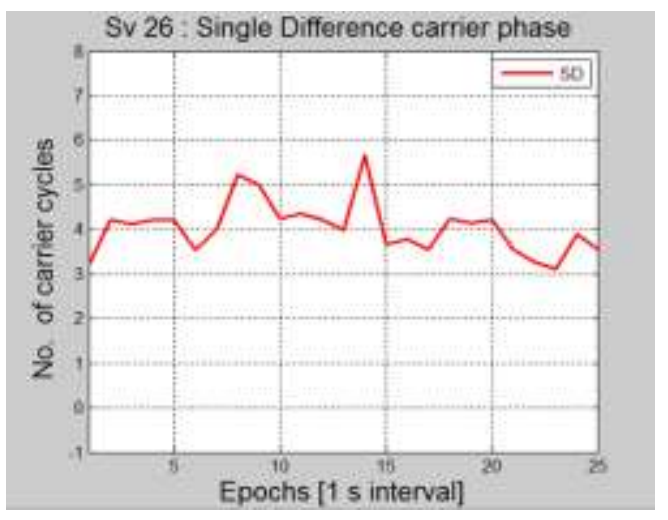

Figure 7. SD between Two Receiver

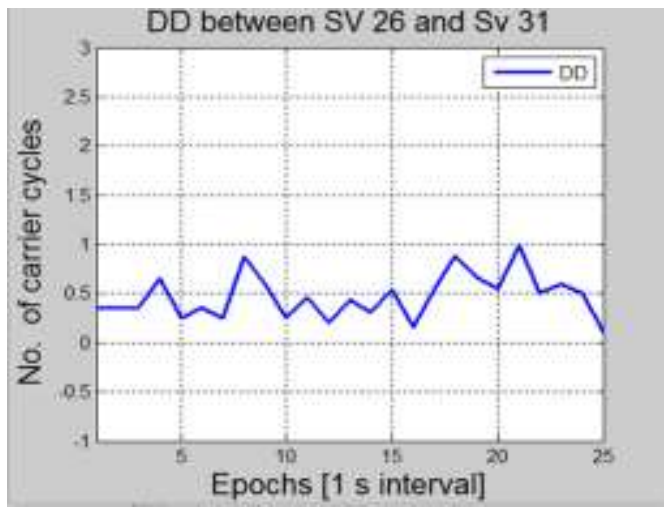

Figure 8. DD Measurement

Figure (7) is the single difference measurement for Sv26, calculated by subtracting the rover measurement (Green line at the bottom) from the base measurement (third line above) in Figure (6). The cancellations that occur from the subtraction that reduced the yaxis scale down from $10^{\wedge} 6$ to just less than tens of cycles. We have canceled the satellite clock errors by the single difference. Now the y-axis becomes small in Figure (7). DD is demonstrated in Figure (8). Figure (8) shows the subtracted two single difference formed by sv 26 and sv 31 or generally, referred to as DD. In this way, we need at least four DDs to solve the unknown parameters. For experiment B the common satellites logged in the RINEX file were ten, out of them the chosen satellites were Sv16, Sv23, Sv26, Sv27, Sv31 with elevation angles $64.0170^{\circ}, 39.0770^{\circ}, 59.1430^{\circ}, 49.7020^{\circ}, 41.3240^{\circ}$ respectively. Out of them, Sv16 was chosen as a reference to form an SD and DD.

\subsection{Baseline and Position of the Rover Receiver Performance}

In Figure (9), the reference baseline can be seen in a straight line in the middle, $0.70 \mathrm{~m}$. Observed baseline length can be seen at in a zig-zag line during 200 epochs. At initial epochs, obtained baseline results are not satisfactory but after 20 epochs expected values are obtained. 


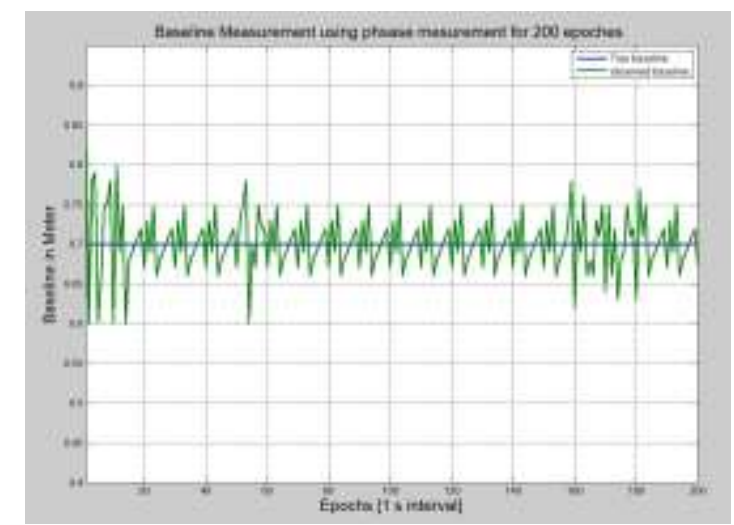

Figure 9. Baseline Determination from Exp. A

The obtained parameters are shown in Table I.

Table 1. Baseline Components from Experiment A

\begin{tabular}{|l|l|l|}
\hline $\begin{array}{l}\text { Baseline } \\
\text { length } \\
\text { Reference }\end{array}$ & $\begin{array}{l}\text { Obtained mean baseline } \\
\text { from 200 epochs }\end{array}$ & $\begin{array}{l}\text { The mean Baseline vector } \boldsymbol{b} \\
\text { obtained from the phase } \\
\text { observation. }\end{array}$ \\
\hline $70 \mathrm{~cm}$ & $73.22 \mathrm{~cm}$ & $\Delta \mathrm{E}($ East $)=0.67 \mathrm{~m}$ \\
& & $\Delta \mathrm{N}(\mathrm{North})=0.3825 \mathrm{~m}$ \\
& & $\Delta \mathrm{U}(\mathrm{Up})=0.01 \mathrm{~m}$ \\
\hline
\end{tabular}

From Table I, those three components (or baseline vectors) are used to compute both the heading of the system and the position of the rover receiver $\boldsymbol{r}$.

We know the approximate position of both the receivers (from the header section of the RINEX observation files). Then the position of the $s$ using pseudo range was computed. Further, the true position for the ' $r$ ' can be found while corrected to the initial position (approximate position) of $r$. The Figure (10) below shows the corrected to initial position using phase observation through baseline observation vector $\boldsymbol{b}$,

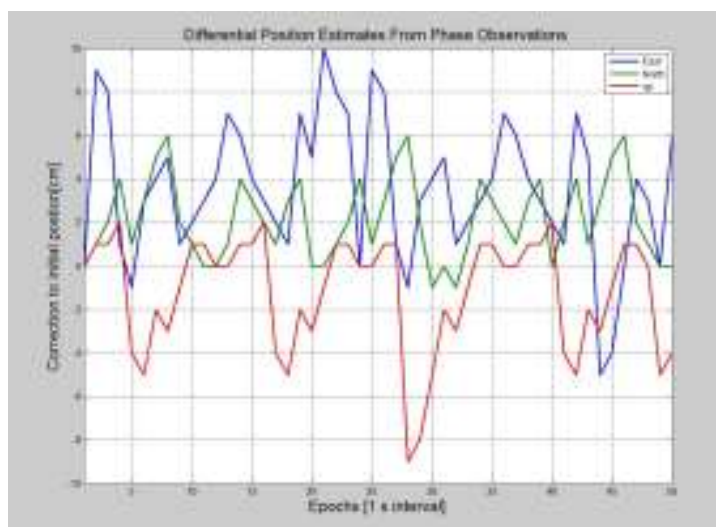

Figure 10. Position of $R$ Estimated from Phase Observations for Set Up A

In above Figure (10) we can notice that the position correction is in $\mathrm{cm}$ level during 50 epochs. The final position of the Experiment A (in ECEF frame) is $X=-3124151.3026$, $\mathrm{Y}=4126723.8754, \mathrm{Z}=3714573.9821$. In this way, for experiment $\mathrm{B}$, the nature of the result appears similar to experiment $\mathrm{A}$. 


\subsection{Heading Angle Performance}

Calculation of heading determination was focused in this research work, although our experiment can calculate two attitude parameters (heading and pitch). The reference heading was obtained from the Google Earth via Internet. The calculated heading angles are shown as follows

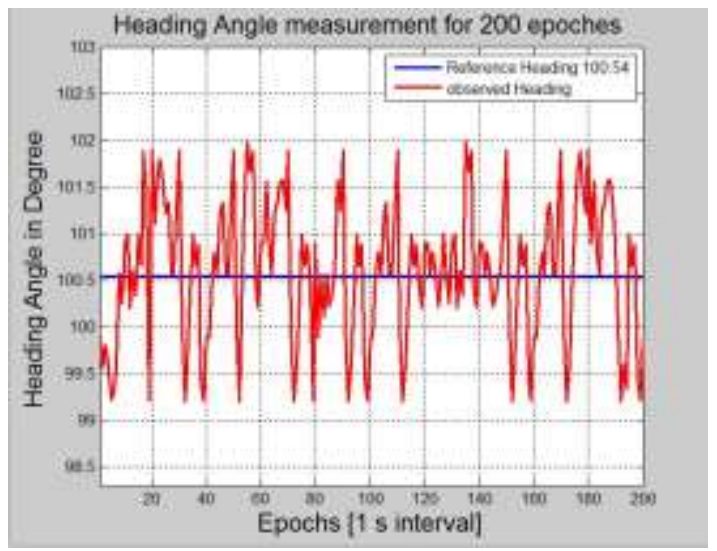

Figure 11. Experiment A, Heading Determination

The above Figure (11) shows the heading calculation during 200 epochs. The RMSE and standard deviation of the Figure (11) are shown in the Table (II).

Table 2. Result Compare to Google Earth

\begin{tabular}{|l|l|l|l|}
\hline $\begin{array}{l}\text { Reference } \\
\text { Heading(Google } \\
\text { Earth })\end{array}$ & $\begin{array}{l}\text { Mean } \\
\text { obtained } \\
\text { Heeding }\end{array}$ & RMSE & Standard Deviation \\
\hline $100.54^{0}$ & $100.6068^{0}$ & $0.0668^{0}$ & $4.986 \mathrm{e}-13$ \\
\hline
\end{tabular}

In this way we followed the experiment $\mathrm{B}$. The Heading angle has been represented by a red line and the reference heading is shown in blue line as shown in Figure (12).

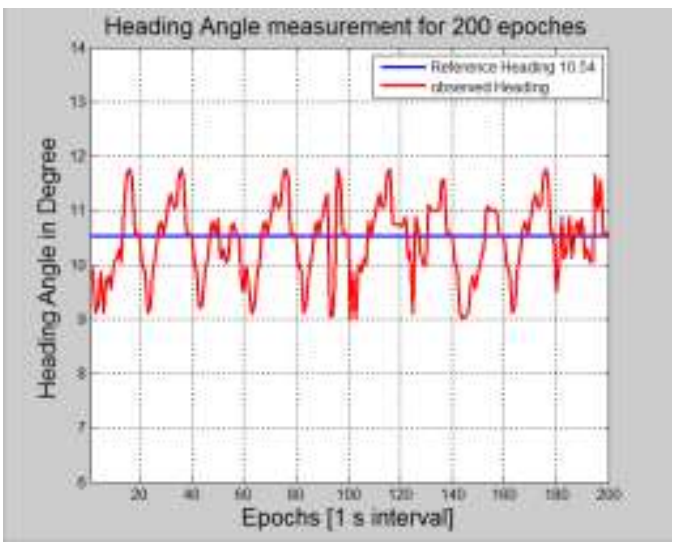

Figure 12. Experiment B, Heading Determination 
In Figure (12), we can notice that the reference heading in blue line and the determined heading is in red line with 1 second of interval. The table below shows the error and standard deviation

Table 3. Result Compare to Google Earth

\begin{tabular}{|l|l|l|l|}
\hline $\begin{array}{l}\text { Reference } \\
\text { Heading } \\
\text { (Google } \\
\text { Earth) }\end{array}$ & $\begin{array}{l}\text { Obtained } \\
\text { Mean } \\
\text { Heading }\end{array}$ & RMSE & Standard Deviation \\
\hline $10.54^{0}$ & $10.43^{0}$ & $0.0705^{0}$ & $2.493 \mathrm{e}-14^{0}$ \\
\hline
\end{tabular}

Table III shows the obtained heading, the RMS error and standard deviation of the platform for experiment B. In this way we can say that the performed experiments for heading accuracy with ultra-short distance baseline are much more suitable for different GPS-related projects such as heading of the vehicles, four wheel robots, quad rotor etc.

\section{Summary and Conclusion}

The heading of the platform between two receivers with a single difference and double difference measurement has been demonstrated. The RINEX files were post-processed in MATLAB environment and RMSE's have been shown for L1 frequency only to make the code compatible with low-cost GPS receivers. The obtained heading of the system has been compared to Google earth to show that the system is suitable for finding a heading with a required accuracy. Elevation mask under 15 degree is ignored during formation of single and double difference to obtain the position accuracy of the satellites. Carrier phase Integer ambiguity cycles have been solved by using the LAMBDA method. The position of the rover receivers has been calculated with respect to the position of the stationary receiver.

The performed experiments A and B proved that the platform of short distance baseline is able to find a required accuracy of heading within 1 degree. For position (relative positioning) of the rover receiver, the accuracies are shown within centimeter level. For future work, the real-time processing and integer ambiguity resolution performance would be taken into account.

\section{Acknowledgement}

This research was financially supported by the Ministry of Trade, Industry and Energy (MOTIE) and Korea Institute for Advancement of Technology (KIAT) through the International Cooperative R\&D program (N046200012).

\section{References}

[1] G. Zheng and D. Egziabher, "Methods for Enhancing Carrier Phase GNSS Positioning And Attitude Determination Performance", (2010).

[2] C. E. Cohen, "Attitude determination using GPS", PhD thesis, Department of Aeronautics and Astronautics, Stanford University, Stanford, CA, (1994).

[3] P. Misra and P. Enge, "Global Positioning System: Signals, Measurements and Perfor-mance", GangaJamuna Press, (2001)

[4] X. W. Chang, C. C. Paige and L. Yin, "Code and Carrier Phase Based Short Baseline GPS positioning Computational Aspects", Mc Gill University, school of Computer science, (2004).

[5] P. J. de Jonge, C. C. J. M. Tiberius and P. J. G. Teunissen, "Computational Aspects of the LAMBDA Method for GPS Ambiguity Resolution”, Geodetic Computing Centre, Delft University of Technology, (1996).

[6] https://www.google.com/earth/explore/products/. 
[7] J. G. Garcia, P. I Mercader and C. H. Muravchik, "Use of carrier phase Double Differences", Latin American applied research, LEICI, (2005).

[8] HEADRT Operators manual (for G12 and GG 24). Sun. H., M.E. Cannon , and G Lachapelle. Department of Geomatics engineering, the University of Calgary. Alberta, Canada, (1997).

[9] https://www.u-blox.com/en/product/u-center-windows.

[10] http://www.rtklib.com/.

[11] W. Gurtner, "The Receiver Independent Exchange format version 3.00", Astronomical Institute University of Bern, (2007).

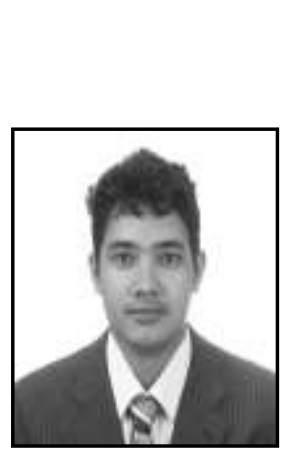

\section{Authors}

Jhabindra Khanal, he was born in Nepal in 1989. He received his Master degrees from Chonbuk National University (CBNU),Jeonju, Korea, in 2016. He is currently doing Ph.D. at CBNU. His research interests include GPS Navigation system, Network control, Robotics, and Neural Network.

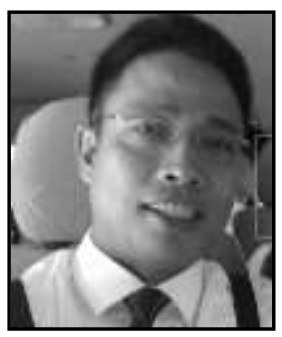

Felipe P. Vista IV, he was born in Phillipines. He received his $\mathrm{Ph} . \mathrm{D}$ degrees from Chonbuk National University(CBNU), Jeonju, korea, in 2014. He is currently working as a researcher(as a postdoctor) at CBNU. His research interests are in the areas of signal processing, GPS, Network system control, Embeded system, and Neural Network.

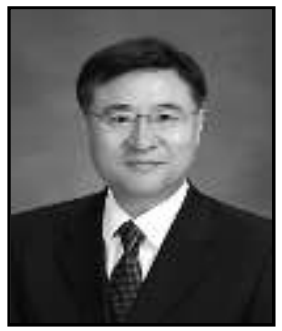

Kil To Chong, (Corresponding author), he received his Ph.D. in Mechanical Engineering from Texas A\&M University in 1995. Currently, he is a professor at the School of Electronics and Information Engineering at Chonbuk National University in Jeonju, Korea, and is head of the Advanced Research Center of Electronics. His research interests are in the areas of signal processing, motor fault detection, network system control, time-delay systems, and Neural Network. 
International Journal of Control and Automation Vol.10, No.6 (2017) 